\title{
Learning about qualitatively different outcomes during a blocking procedure
}

\author{
ROBERT A. RESCORLA \\ University of Pennsylvania, Philadelphia, Pennsylvania
}

\begin{abstract}
In four experiments, rat subjects were used in appetitive Pavlovian magazine-approach and instrumental conditioning procedures. Experiments 1 and 2 found successful blocking of the Pavlovian conditioning of $\mathrm{X}$ when it was reinforced in an $\mathrm{AX}$ compound after prior conditioning of $\mathrm{A}$. This occurred whether the outcome following AX was the same as or qualitatively different from what followed A. Experiment 3 repeated those findings but also used a transfer procedure to identify the individual associations between $\mathrm{X}$ and outcomes. Stimulus $\mathrm{X}$ developed an association with the outcome following $\mathrm{AX}$ when that outcome differed from that following $\mathrm{A}$ alone but not when it was the same as that following A alone. Experiment 4 repeated that pattern of observations for the case of an $\mathrm{X}$ that was an instrumental discriminative stimulus. These results suggest that different associative structures may result from a qualitatively changed and unchanged outcome in a blocking experiment. The results are related to comparable findings for the case of overexpectation.
\end{abstract}

Few Pavlovian conditioning phenomena have attracted more attention in the last several decades than has blocking. The well-documented ability of a previously conditioned stimulus (A) to block conditioning of another stimulus $(\mathrm{X})$ on reinforced $\mathrm{AX}$ trials has been widely studied in many conditioning preparations (Kamin, 1968; see Rescorla \& Holland, 1982), and it has had a profound effect on theorizing. Most authors have interpreted these results in terms of differential learning about $X$, although some (e.g., Miller \& Matzel, 1988) have attributed them to differential performance.

One reason for the continuing interest in blocking is that it implies some complexity in the conditions that produce associative learning. That complexity is frequently described in terms of the importance of the information that a stimulus $\mathrm{X}$ provides about the reinforcing outcome $(\mathrm{O})$ in determining the development of the $\mathrm{X}-\mathrm{O}$ association. It is common to describe the $\mathrm{A}$ stimulus as making $\mathrm{X}$ redundant, thereby depriving it of informativeness and hence of conditioning.

A natural implication of this description is that conditioning of $\mathrm{X}$ might occur on the AX trials if steps were taken to ensure X's predictiveness. For instance, if the nature of the outcome were changed at the time when $\mathrm{X}$ began to accompany $\mathrm{A}$, that might make $\mathrm{X}$ informative and hence allow it to become conditioned. There is certainly evidence that increasing the intensity or frequency of the outcome on the AX trials, compared with that used on the A-alone trials, will allow X to develop conditioning (e.g., Dickinson, Hall, \& Mackintosh, 1976; Holland,

This research was supported by National Science Foundation Grant IBN94-04676. Correspondence concerning this article should be addressed to R. A. Rescorla, Department of Psychology, University of Pennsylvania, 3815 Walnut Street, Philadelphia, PA 19104 (e-mail rescorla@cattell.psych.upenn.edu).
1984). The consequences of reducing the intensity or frequency on the introduction of $\mathrm{X}$ have been more varied. Some have found it to allow X to develop excitatory conditioning (e.g., Dickinson et al., 1976) whereas others have reported that it encourages $\mathrm{X}$ to become inhibitory (e.g., Cotton, Goodall, \& Mackintosh, 1982; Wagner, Mazur, Donegan, \& Pfautz, 1980). Holland (1988) has offered some results that may help resolve these conflicts.

A related case of particular interest is that in which the outcome changes in terms of its qualitative properties but not in terms of its intensity or frequency. Despite occasional reports that these changes disrupt blocking (e.g., Blaisdell, Denniston, \& Miller, 1997), many authors have reported that they can leave blocking undisturbed (e.g., Bakal, Johnson, \& Rescorla, 1974; Ganesan \& Pearce, 1988; Williams, 1994). Such a result does damage to the informal notion that the information value of the stimulus is important in determining conditioning. It is particularly damaging when accompanied by demonstrations that the differences among outcome identities are readily detected by the animal (e.g., Williams, 1994).

However, this finding seems puzzling in the light of some recent results from Pavlovian conditioning and instrumental learning experiments in which a single stimulus is first followed by one outcome (O1) and then by another, equally valuable, outcome (O2) (e.g., Delamater, 1996; Rescorla, 1992, 1996). Although such procedures produce little enhancement of responding to the stimulus, they can be shown both to allow preservation of the association with $\mathrm{Ol}$ and also to result in the development of a robust association with $\mathrm{O} 2$. That is, prior training of $\mathrm{A}-\mathrm{O} 1$ does not prevent subsequent development of an $\mathrm{A}-\mathrm{O} 2$ association when $\mathrm{A}$ is paired with $\mathrm{O} 2$. However, if prior A-O1 training does not block the conditioning of $\mathrm{O} 2$ to $\mathrm{A}$ itself, it seems surprising that this training nevertheless blocks the conditioning of $\mathrm{O} 2$ to a concurrently present $\mathrm{X}$. 
It is possible that recent results from summation and overexpectation experiments may shed some light on the interpretation of these findings (e.g., Rescorla, 1999). Those experiments used a Pavlovian magazine-approach situation in which rats were conditioned with different stimuli signaling the delivery of various qualitatively different outcomes. They found good summation of responding to stimulus compounds composed of elements that signaled either the same or different outcomes (see, also, Watt \& Honey, 1997). Moreover, reinforcing the compound with one of those outcomes resulted in "overexpectation," depression of subsequently tested performance to the elements of the compound. This depression occurred whether the elements had separately predicted the same or qualitatively different outcomes (see also Ganesan \& Pearce, 1988). Apparently, the two qualitatively different outcomes functioned as though they were the same, despite clear evidence elsewhere that the animal routinely discriminated them (e.g., Colwill \& Rescorla, 1988).

However, more detailed assessment of the underlying associative structures resulting from these experiments suggested that this reduction in performance was not produced by modifications in the associations of the elements with the outcome. A transfer test assessed the status of the various element-outcome associations, in terms of the ability of the element to control performance of a response that has earned the same outcome. That test found the element-outcome associations to be of similar strength whether or not the element had been subjected to an overexpectation procedure. The preservation of the element-outcome association at a time when there is a depression of performance suggests the presence of some other, outcome-independent, depressive process. For instance, it might be that some response-specific process develops as a result of this overexpectation procedure (Rescorla, 1993, 1998).

One might suppose that a related process functions in the course of a blocking experiment in which the outcome identity is changed. In a situation in which first $\mathrm{A}$ is conditioned by $\mathrm{O} 1$ and then $\mathrm{AX}$ is conditioned by $\mathrm{O} 2$, the lack of performance to $X$ may not indicate the failure of an $\mathrm{X}-\mathrm{O} 2$ association to develop; rather, it may mean that the acquisition of an $\mathrm{X}-\mathrm{O} 2$ association is accompanied by the concurrent acquisition of inhibitory learning. Indeed, the dynamics of these changes might involve the same mechanisms as those that occur in overexpectation. When the AX compound is reinforced by $\mathrm{O} 2$, both $\mathrm{A}$ and $\mathrm{X}$ may begin to develop an association with $\mathrm{O} 2$, but $\mathrm{A}$ may also retain its original association with $\mathrm{O} 1$. As a result, the AX compound may become composed of two stimuli whose total summed associative strength is too large to be appropriate to the outcome $(\mathrm{O} 2)$ actually occurring. Consequently, reinforcing the compound with $\mathrm{O} 2 \mathrm{might}$ result in overexpectation, the conditioning of a depressive process to both A and X. Asymptotically, one would expect $A$ and $X$ each to have both a moderately strong association with $\mathrm{O} 2$ and also a sufficiently strong outcomeindependent inhibitory association to counterbalance that
$\mathrm{O} 2$ association. The result would be that despite the failure of $\mathrm{A}$ to block $\mathrm{X}$ from developing an $\mathrm{O} 2$ association (or even to prevent its own development of association with $\mathrm{O} 2$ ), nevertheless there is little enhancement of responding to $X$.

According to this account, the failure to observe conditioning to $\mathrm{X}$ in the case of a changed outcome does not reflect actual blocking of the $\mathrm{X}-\mathrm{O} 2$ association but, rather, a more complex set of results in which that learning is masked. This can be contrasted with the case in which the outcome identity is unchanged at the time that $X$ is introduced; in that case, low performance to X might represent the ability of A to prevent conditioning of $\mathrm{X}$ by the outcome following AX.

The present experiments were designed to assess the merits of this account, using a preparation in which outcomes known to be discriminably different nevertheless produce summation and overexpectation. Experiments 1 and 2 documented blocking within an appetitive magazine-approach situation for rats, using liquid sucrose and pellets as the outcomes. In Experiment 1, a single outcome was used throughout; in Experiment 2, different outcomes were used for the conditioning of $\mathrm{A}$ and $\mathrm{AX}$. In Experiment 3, a transfer procedure was used to compare the state of the $\mathrm{X}-\mathrm{O}$ associations after blocking with a changed and unchanged outcome. In Experiment 4, the design of Experiment 3 was replicated in a different conditioning preparation, discriminative instrumental responding.

\section{EXPERIMENT 1}

The intention of this experiment was to provide a wellcontrolled demonstration of blocking in the present magazine-approach situation, preparatory to doing the primary analysis. The essential feature of a blocking design is that prior conditioning of $\mathrm{A}$ acts to diminish the conditioning of $\mathrm{X}$ that occurs on reinforced AX trials. In demonstrating blocking, therefore, it is important to document that A's ability to block conditioning to $\mathrm{X}$ is attributable to A's own association with the outcome rather than to a variety of other factors. One can go some distance toward efficiently ruling out many alternative interpretations using the within-subjects design sketched in Figure 1. In that design, two stimuli, $\mathrm{X}$ and $\mathrm{Y}$, both receive conditioning in compound with two other stimuli $A$ and $\mathrm{B}$. However, $\mathrm{A}$ and $\mathrm{B}$ differ in that $\mathrm{A}$ has a history of pairing with the outcome and $B$ does not. If prior conditioning of $A$ allows it to block conditioning of $X$, subsequent testing should show a greater response to $Y$ than to $\mathrm{X}$. The fact that both $\mathrm{X}$ and $\mathrm{Y}$ receive conditioning in the presence of other stimuli allows one to rule out the simple presence of another stimulus during X's conditioning (i.e., overshadowing) as the operative factor. The fact that the same animal has a history of exposure to the individual $\mathrm{A}$, $\mathrm{B}$, and the outcome events, but with only A signaling the outcome, allows one to attribute any difference in responding during the test of $X$ and $Y$ to the difference in 


\begin{tabular}{l|ll|c} 
Condit & \multicolumn{2}{|c|}{ Compound } & Test \\
\hline A-01 & A-01 & AX-01 & $X$ \\
B- & B- & BY-01 & $Y$
\end{tabular}

Figure 1. Design of Experiment 1. Discriminative conditioning of two visual stimuli ( $A$ and $B$ ) was followed by reinforcement of each in compound with an auditory stimulus ( $X$ or $Y$ ). The auditory stimuli were tested for responding.

conditioning of $\mathrm{A}$ and $\mathrm{B}$ rather than to simple exposure to the individual events themselves. Several previous investigators have put such a design to good use in demonstrating blocking (e.g., Betts, Brandon, \& Wagner, 1996; Wagner, 1976).

In the present experiment, a solid pellet was used as the outcome and the response measured was approach to the location of its delivery. The A and B were visual stimuli that were either paired or unpaired with that pellet. The $\mathrm{X}$ and $\mathrm{Y}$ were auditory stimuli.

\section{Method \\ Subjects and Apparatus}

The subjects were 16 male Sprague-Dawley rats about 90 days old. They were housed in individual cages and maintained on a food-deprivation regime that kept them at $80 \%$ of their ad-lib body weight. They had free access to water in the home cage.

The apparatus consisted of four operant chambers, measuring $22.9 \times 20.3 \times 20.3 \mathrm{~cm}$, identical to those used in previous reports (e.g., Colwill \& Rescorla, 1985). The two end walls of each chamber were aluminum; the side walls and ceiling were clear Plexiglas. The floor of the chamber was composed of $0.48-\mathrm{cm}$ stainless steel rods spaced $1.9 \mathrm{~cm}$ apart center to center. Each chamber had a recessed food magazine in the center of one end wall. Two small metal cups measuring $1.25 \mathrm{~cm}$ in diameter and $1.5 \mathrm{~cm}$ deep were sunk side by side in the floor of each food magazine. An infrared detector and emitter system was mounted on the side walls of the magazine, permitting automatic recording of head movements into the magazine. To the left of the magazine was a lever; to the right was a chain suspended from a microswitch mounted on the lid of the chamber. Located directly above the food magazine was a $2-\mathrm{cm}$ opening behind which was an aluminum plate that activated an attached microswitch when displaced by a nosepoke. During Pavlovian conditioning procedures, access to these manipulanda was blocked by covering the lever with a metal shield, retracting the chain through a hole in the ceiling, and covering the nosepoke opening with a jeweled lens. These manipulanda were not used in Experiments 1 and 2, but were used in the related Experiments 3 and 4 .

Each chamber was enclosed in a sound- and light-resistant shell. Mounted on the inside wall of this shell were speakers that permitted the presentation of a white noise $(\mathrm{N})$ and an $1800-\mathrm{Hz}$ tone $(\mathrm{T})$, each measuring approximately $76 \mathrm{~dB}$ re $20 \mu \mathrm{N} / \mathrm{m}^{2}$ against a background level of $62 \mathrm{~dB}$ (C scale). Also mounted on that wall was a relay that could be pulsed at a rate of $2 / \mathrm{sec}$ to produce a clicker (C) and a 6 -W bulb that could be illuminated to provide a light (L) stimulus during the otherwise dark session. Another 6-W light was mounted on the ceiling of the chamber; this light could be pulsed on at a rate of $1 / \mathrm{sec}$ to produce a flashing $(\mathrm{F})$ stimulus. The outside ceiling of the shell supported a solenoid-operated gravity feed valve connected via plastic tubing to the leftmost cup in the food maga- zine. That system permitted the presentation of $.3 \mathrm{ml}$ of an $8 \%$ sucrose solution. Also attached to that food magazine was a dispenser containing 45-mg pellets (P. J. Noyes Co., Formula A).

Experimental events were controlled and recorded automatically by relays and microprocessors located in an adjoining room.

\section{Procedure}

Magazine training. On the first day, the animals received a 20 min magazine training session, during which 20 noncontingent deliveries of pellets were given at time intervals variable around a mean of $1 \mathrm{~min}$.

Pavlovian conditioning. On each of the next 12 days, all animals received Pavlovian discriminative conditioning with the two visual stimuli, $L$ and F. Each session contained sixteen 30 -sec presentations of each stimulus. For half of the animals, $L$ ended in a pellet and $\mathrm{F}$ was nonreinforced; for the remaining half of the animals, the contingencies were reversed. The intertrial interval (ITI), measured between trial initiations, was variable around a mean of $2.5 \mathrm{~min}$, resulting in a session duration of $80 \mathrm{~min}$.

Preexposure. On the next day, all animals received nonreinforced preexposure to the two auditory stimuli, $\mathrm{N}$ and $\mathrm{C}$. That session contained eight 30 -sec presentations of each stimulus, delivered with a mean ITI of $2.5 \mathrm{~min}$, yielding a session duration of $40 \mathrm{~min}$. No pellets were given during this session.

Compound conditioning. On each of the next 4 days, the animals received compound presentations of the auditory and visual stimuli. In a manner counterbalanced with regard to the previous treatments, half of the animals received compounds of LN and FC; the other half received the alternative compounds of $\mathrm{LC}$ and $\mathrm{FN}$. All eight 30 -sec presentations of each of the compounds terminated in pellets. In addition, to preserve the differential associative strengths of $L$ and $F$, each session also contained eight 30 -sec presentations of each of the visual stimuli alone, with the differential reinforcement contingencies of initial conditioning retained. The 2.5-min ITI generated a session duration of $80 \mathrm{~min}$.

Test. On the next day, the animals received a single test session. The first portion of that session consisted of a half session of compound conditioning. The remainder consisted of four $30-\mathrm{sec}$ nonreinforced presentations each of $\mathrm{N}$ and $\mathrm{C}$. The average ITI remained $2.5 \mathrm{~min}$, yielding a session duration of $60 \mathrm{~min}$. The data of primary interest are the response rates during those test presentations of $\mathrm{N}$ and $\mathrm{C}$, as a function of whether or not the visual stimulus in whose presence they had been reinforced had itself received prior conditioning.

\section{Results and Discussion}

Figure 2 shows the results of the discrimination learning with the visual stimuli and the results of compound conditioning. That figure displays responding during the $30 \mathrm{sec}$ prior to any stimulus presentation and during each of the differentially treated visual stimuli. It is clear that the discrimination posed no difficulties, with the animals rapidly showing differential performance to the reinforced and nonreinforced visual stimuli. This differential performance was initially preserved in the presence of the compounds containing the auditory stimuli. On the first day of compound conditioning, responding was reliably greater to the AX compound (which contained the separately reinforced visual $A$ ) than to the BY compound (which contained the separately nonreinforced visual B) [Wilcoxon $T(16)=0, p<.01$ ]. However, responding to BY grew rapidly over days, so that responding to the compounds was comparable at the end of this phase. During this phase of the experiment, the separate differential 


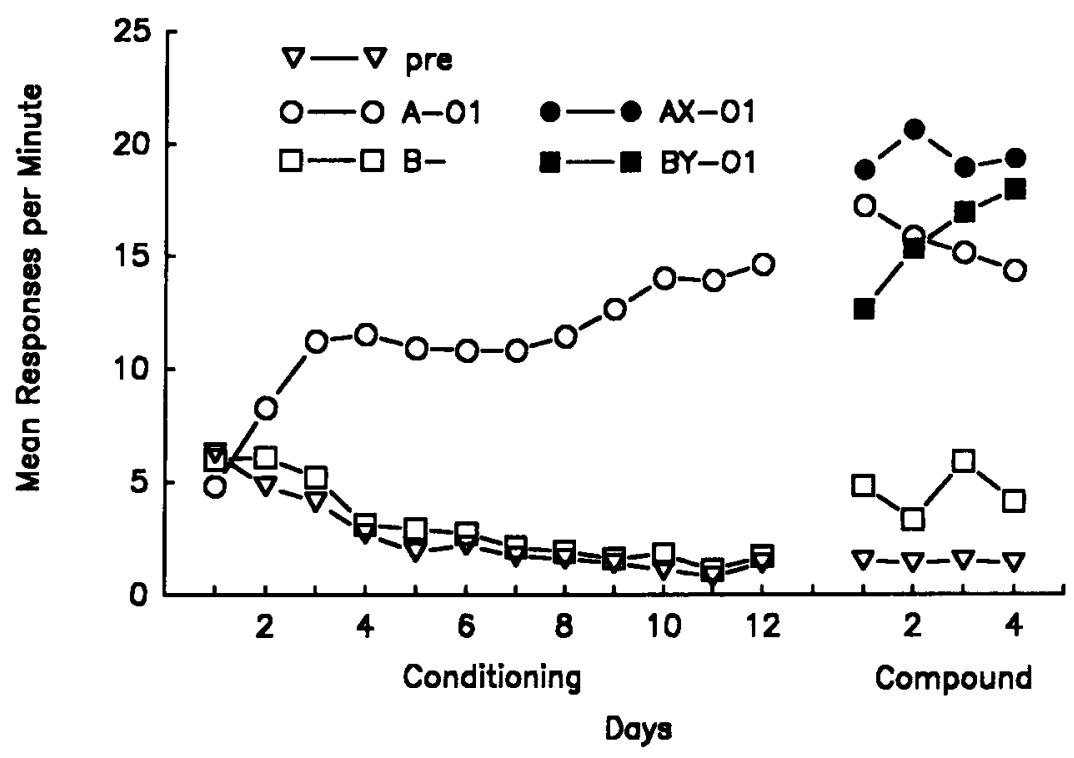

Figure 2. Results of initial conditioning and compound training for Experiment 1. Responding is shown during the prestimulus period, during the reinforced $A$ and nonreinforced $B$, and during the reinforced $A X$ and $B Y$ compounds. The reinforcer was the pellet outcome (O1). The stimuli $A$ and $B$ were a steady and flashing light, counterbalanced; $X$ and $Y$ were a noise and clicker, counterbalanced.

treatment of the visual stimuli served to maintain their discrimination.

The data of most interest, from the final test of the auditory $\mathrm{X}$ and $\mathrm{Y}$ elements, are shown in Figure 3. That figure displays responding on individual trials for the period prior to stimulus onset and then during the $\mathrm{X}$ and $\mathrm{Y}$ stimuli. It is clear that both stimuli augmented responding somewhat, but that the augmentation was substantially higher for the control Y stimulus than for the blocked X stimulus. The difference was especially large on the initial trials but persisted throughout the nonreinforced test. For the test session as a whole, $Y$ showed reliably greater responding than $\operatorname{did} \mathrm{X}[T(15)=15, p<.01 ; S E M \mathrm{~s}=0.36$ and 0.69 , respectively].

These data show clear evidence of blocking in the present magazine-approach preparation. Responding to $X$ and $\mathrm{Y}$ was differential, depending on the prior differential conditioning of the A and B element in whose presence they had been reinforced.

\section{EXPERIMENT 2}

This experiment was a replication of Experiment 1 except that the outcome delivered after the AX and BY compounds was qualitatively different from that used in the discrimination training of $\mathrm{A}$ and $\mathrm{B}$. The two outcomes used were the solid pellet employed in Experiment 1 and an $8 \%$ liquid sucrose. These are known from prior work (e.g., Colwill \& Rescorla, 1985) to be of comparable value but to be easily discriminated from each other. The question was whether interchanging them in the course of a blocking experiment would nevertheless allow blocking to occur.

\section{Method}

\section{Subjects and Apparatus}

The subjects were 16 naive rats of the same sort as those used in Experiment 1; they were maintained in the same manner as in Experiment 1 . The apparatus was that used in Experiment 1.

\section{Procedure}

The procedure was identical to that of Experiment 1 with two exceptions. First, the animals received two magazine training sessions, one with pellet and the other with sucrose outcomes. Second, during initial conditioning, half of the animals received sucrose as the reinforcing outcome and half received pellets. In the compound phase of the experiment, the compounds were followed in every case by the outcome not used in initial conditioning of the visual stimuli. As in Experiment 1, the compound phase also included separate presentations of the visual elements each followed by the same outcome as that used in initial conditioning, in order to maintain their value.

\section{Results and Discussion}

The results were quite similar to those of Experiment 1 . Figure 4 shows the course of initial acquisition and compound conditioning. The discrimination between visual stimuli developed rapidly. On the final day of simple conditioning, the mean responses per minute were 11.5 and 13.5 during the stimulus reinforced with pellets and sucrose, respectively. Initial responding differed during the compounds containing reinforced and nonreinforced visual stimuli. As in Experiment 1, responding on the first day of compound training was greater to the 


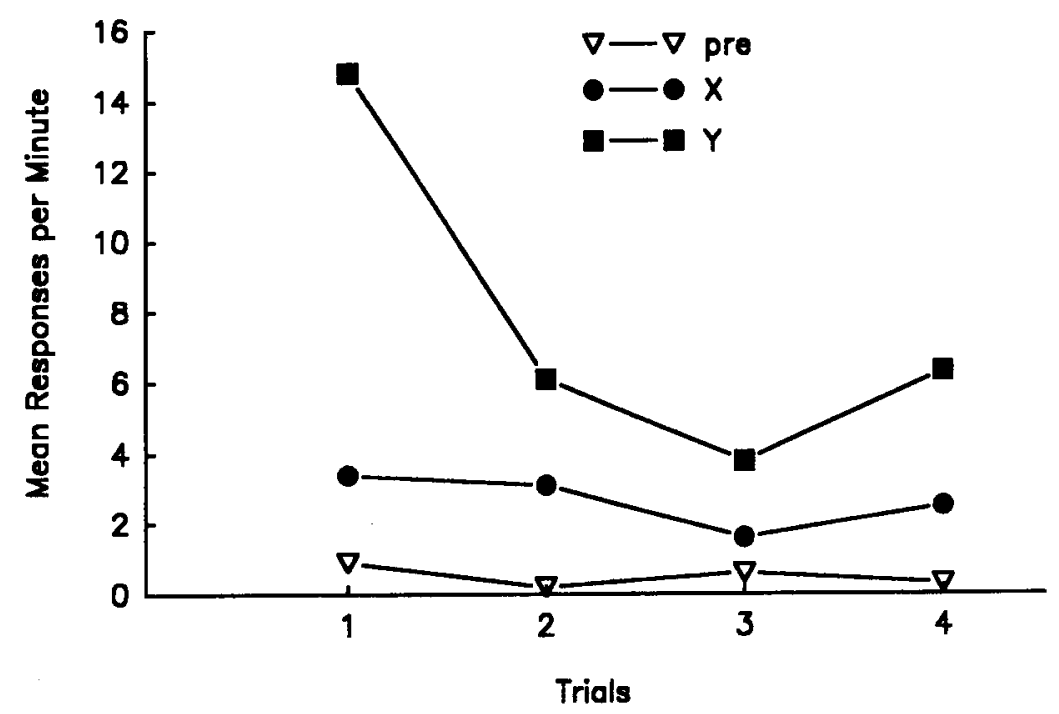

Figure 3. Test results for Experiment 1. Responding is shown for the prestimulus period-and during the control $(Y)$ and blocked (X) stimuli. Stimulus identifications are the same as in Figure 2.

AX than to the BY compound $[T(16)=0, p<.01]$. However, that difference was attenuated by the end of compound conditioning.

Figure 5 shows the results of the test session in which the auditory $\mathrm{X}$ and $\mathrm{Y}$ stimuli were separately presented without additional reinforcement. As in Experiment 1, both stimuli augmented responding relative to the prestimulus period; however, as in that experiment, the augmentation was substantially greater during the control $\mathrm{Y}$ stimulus than during the blocked $\mathrm{X}$ stimulus. Over the test session as a whole, the difference proved reliable $[T(13)=$ $15, p<.05 ; S E M \mathrm{~s}=0.34,0.53]$. These results suggest

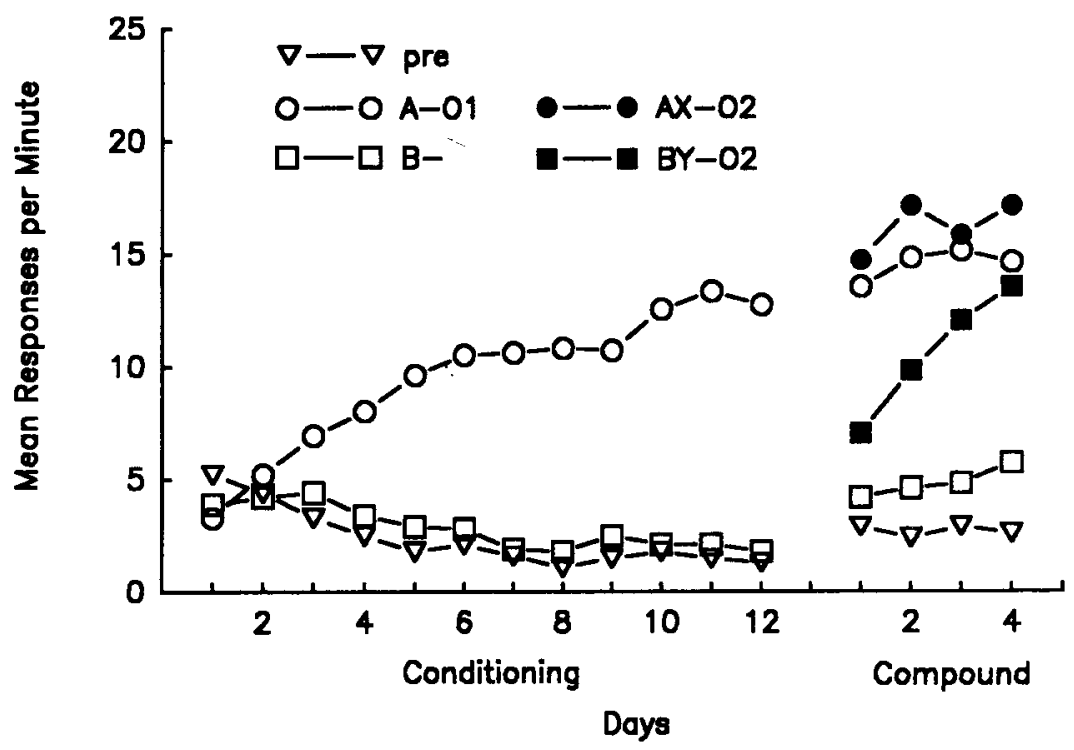

Figure 4. Results of initial conditioning and compound training for Experiment 2. Responding is shown during the prestimulus period, during the reinforced $A$ and nonreinforced $B$, and during the reinforced $A X$ and $B Y$ compounds. The reinforcer was either a pellet or sucrose outcome (O1) in phase 1 and the alternative in phase 2 (O2). Stimulus identifications are the same as in Figure 2. 


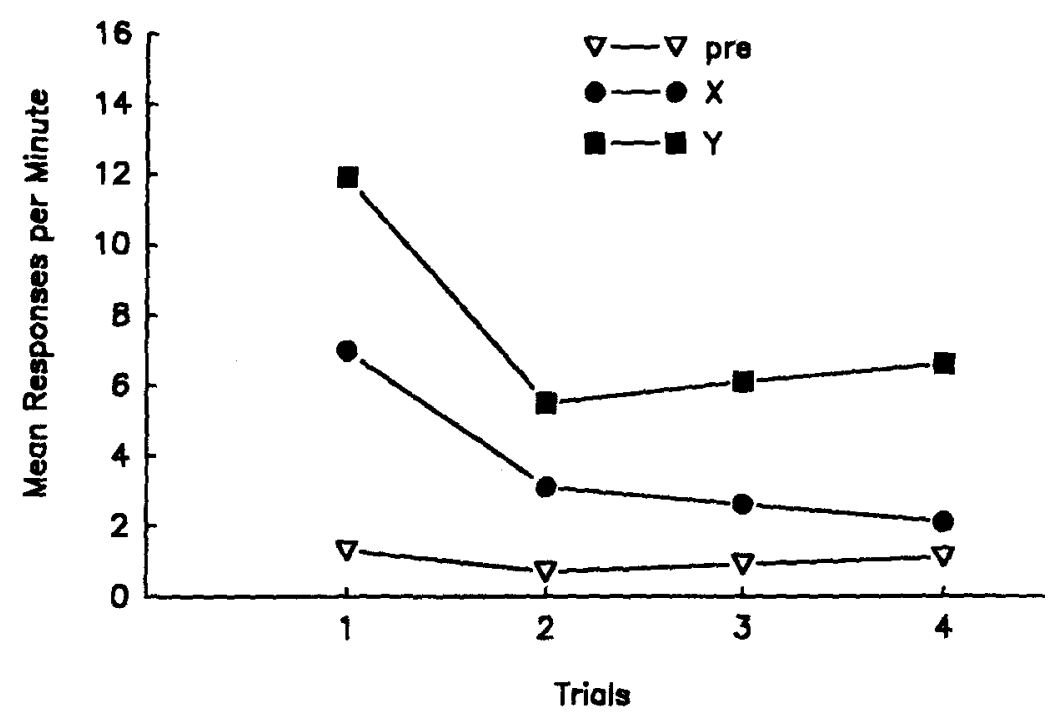

Figure 5. Test results for Experiment 2. Responding is shown for the prestimulus period and during the control $(Y)$ and blocked $(X)$ stimuli. The $X$ and $Y$ were a clicker and noise, counterbalanced.

that substantial blocking of $X$ occurred despite the fact that the outcome paired with the AX compound was qualitatively different from what was paired with $\mathrm{A}$.

There is the suggestion in Figures 3 and 5 that blocking may have been less complete in Experiment 2 than in Experiment 1 . However, a comparison of the differences between experimental and control stimuli found no reliable differences between Experiments 1 and 2 on either Trial 1 [Mann-Whitney $U(16,16)=87, S E M \mathrm{~s}=1.74,1.22]$ or for the session as a whole $[U(16,16)=103, S E M s=0.65$, 0.81 ]. Of course, cross-experiment comparisons of this sort are always hazardous, especially in cases such as this where the range of outcomes experienced is different. Experiments 3 and 4 provide more appropriate circumstances under which to compare directly the responses to an $X$ stimulus reinforced with a qualitatively changed or unchanged outcome.

\section{EXPERIMENT 3}

The observation of successful blocking in both Experiment 1 and Experiment 2 raises two questions. First, is the magnitude of blocking comparable whether or not the outcome is changed? In the present experiment, this question was addressed by comparing conditioning to two stimuli, $X$ and $Y$, as a result of reinforced $A X$ and $B Y$ trials, when the reinforcer used following $A X$ was the same as that following $A$ alone but the reinforcer used following BY was different from that following $B$ alone. Second, are the underlying associative changes the same in both cases? This question was addressed by assessing the magnitude of the individual associations that $\mathrm{X}$ and $Y$ have with their outcomes, using a transfer design in which the stimuli were asked to control instrumental responses which had earned the various outcomes. The design is sketched in Figure 6.

Initially, all animals received training in which two instrumental responses, a leverpress and a chainpull (counterbalanced as $R 1$ and R2), earned different outcomes, pellets and sucrose (counterbalanced as $\mathrm{O} 1$ and $\mathrm{O} 2$ ). Then all animals received initial Pavlovian conditioning in which the two visual stimuli (A and $B$ ) were conditioned using those different outcomes. That conditioning was followed by a phase of compound conditioning in which

\begin{tabular}{l|c|c|c|c} 
Response & Condit & \multicolumn{2}{|c|}{ Compound } & \multicolumn{2}{|c}{ Test } \\
\hline$R 1-01$ & $A-01$ & $A-01 \quad A X-01$ & $X$ & R1 V R2 \\
$R 2-02$ & $B-02$ & $B-02$ & $B Y-01$ & $Y$
\end{tabular}

Figure 6. Design of Experiment 3. After initial training of instrumental responding on the lever and chain $(R 1$ and $R 2)$ with the differential outcomes (O1 and $\mathrm{O2}$ ), all animals received Pavlovian conditioning of two visual stimuli ( $A$ and $B$ ) with those outcomes. Then compound $A X$ and $B Y$ trials were added, both receiving one outcome. Finally, $X$ and $Y$ were tested for their ability to transfer control to the lever and chain. Stimulus identifications are the same as in Figure 4. 
the visual stimuli were joined by the auditory stimuli ( $\mathrm{X}$ and $Y$ ) and the compounds both reinforced either by $\mathrm{O} 1$ or by $\mathrm{O} 2$. The design was such that the same outcome followed A and AX but different outcomes followed B and BY. Finally, in a test session, the auditory stimuli were presented at a time when the animals had the opportunity to engage in R1 and R2. It is well documented in earlier work that a stimulus will differentially augment an instrumental response to the degree that the stimulus and response share associations with the same outcome (e.g., Colwill \& Rescorla, 1988). Hence, one can use the success of this transfer as an assessment of the associative strengths of the auditory stimuli with the outcomes that reinforced their compounds.

During this test session, it was also important to obtain an index of simple responding comparable to that obtained in Experiments 1 and 2. For this purpose, measurements were taken of the ability of the auditory stimuli not only to transfer to the instrumental responses but also to elicit the Pavlovian conditioned magazine behavior. Earlier work in highly similar procedures has suggested that one can concurrently obtain such measures at the time that transfer is being assessed (Delamater, 1996; Rescorla, 1999).

Consequently, one can compare the results of magazine approach to the two auditory stimuli, $\mathrm{X}$ and $\mathrm{Y}$, in order to assess the magnitude of conditioning of the auditory stimuli whether the outcome has been changed or not. More importantly, one can use the successful transfer of $\mathrm{X}$ and $\mathrm{Y}$ to $\mathrm{R} 1$ and $\mathrm{R} 2$ to assess the strength of their individual associations with the outcomes. If the present account has merit, then $\mathrm{X}$ and $\mathrm{Y}$ should develop different associations with their outcomes, despite showing comparable performance of the magazine-approach response.

\section{Method}

\section{Subjects and Apparatus}

The subjects were 16 naive rats of the same sort and maintained in the same manner as in Experiment 1. The apparatus was that of Experiment 1.

\section{Procedure}

Response training. On each of the first 2 days, the animals received a 20 -min magazine training session. The first session contained 20 pellets; the second contained 20 sucrose deliveries. Over the next 2 days, all animals were trained in separate sessions to leverpress and chainpull. Each training session allowed responding to earn 25 deliveries of an outcome on a continuous reinforcement schedule. For half of the animals, leverpressing led to a pellet and chainpulling to sucrose; for the other half of the animals, the response-outcome relations were interchanged. On each of the next 5 days, all animals received variable-interval (VI) training with the lever and chain. Each day contained two 20 -min sessions, one with lever and one with chain, spaced about $1 \mathrm{~h}$ apart. During each session, responding was reinforced on a VI 1-min schedule, using the reinforcer employed during initial training.

Pavlovian conditioning. On each of the next 12 days the animals received Pavlovian conditioning of $L$ and $F$ in the manner of previous experiments. Each session contained 16 deliveries of each $30-\mathrm{sec}$ stimulus with a mean intertrial interval of $2.5 \mathrm{~min}$, yielding a session duration of $80 \mathrm{~min}$. For each animal, one stimulus termi- nated in a pellet and the other terminated in sucrose, with the contingencies balanced across animals.

Transfer test preparation. Between Days 11 and 12 of Pavlovian conditioning, the animals received 2 retraining days on the instrumental responses. On the first of these, they received two VI sessions in the manner of prior training. On the second day, they received an 8-min choice session in which both lever and chain were present but not rewarded. Prior work has shown such sessions to equalize the rates of the two responses and to enhance their sensitivity to transfer.

Preexposure. On the day following the last conditioning session, all animals received nonreinforced preexposure to the two auditory stimuli, $\mathrm{N}$ and $\mathrm{C}$. That session contained eight 30 -sec presentations of each stimulus, delivered with a mean ITI of $2.5 \mathrm{~min}$. No outcomes were given during this $40-\mathrm{min}$ session.

Compound conditioning. On each of the next 4 days, the animals received compound presentations of the auditory and visual stimuli. In a manner counterbalanced with regard to the previous treatments, half of the animals received compounds of $\mathrm{LN}$ and $\mathrm{FC}$ and the other half received the alternative compounds of $\mathrm{LC}$ and FN. For any particular animal, all eight $30-\mathrm{sec}$ presentations of each of the compounds terminated in the same outcome, with the outcome identity counterbalanced across animals. In addition, in order to preserve the differential associative strengths of $\mathrm{L}$ and $\mathrm{F}$, each session also contained eight presentations of each of the visual stimuli alone, with differential reinforcement contingencies of initial conditioning retained. The result of these treatments was that one auditory stimulus was reinforced by the same outcome as that signaled by its accompanying visual stimulus when it was presented alone; the other auditory stimulus received a different outcome from that which its accompanying visual stimulus separately signaled. The ITI was $2.5 \mathrm{~min}$, yielding a session duration of $80 \mathrm{~min}$.

Test. On the next day, the animals received an 8-min test session in which the auditory stimuli were presented while both the lever and chain were available. The session contained four 30 -sec presentations each of $\mathrm{N}$ and $\mathrm{C}$, in counterbalanced order, delivered with an ITI of $30 \mathrm{sec}$. The results of primary interest are the rates of responding on these manipulanda during $\mathrm{N}$ and $\mathrm{C}$. In addition, the rate of magazine responding was recorded during the prestimulus and stimulus periods.

\section{Results and Discussion}

Initial instrumental response learning and Pavlovian conditioning proceeded as expected. By the final day of VI training, the mean rate of making the instrumental response was $11.5 / \mathrm{min}$, with no reliable differences as a function of the identity of the manipulandum or the outcome. On the final day of Pavlovian conditioning, the mean rate of magazine entry was 16.2 and 16.4 responses/ min during the visual stimuli signaling pellets and sucrose, respectively. Over the course of compound conditioning, responding to the compounds was similar for the compounds that signaling the changed and unchanged outcomes. The rates averaged 18.0 responses $/ \mathrm{min}$ on the last day of compound conditioning.

During the test, both stimuli elevated the rate of magazine entry somewhat over the prestimulus rate of $4.3 / \mathrm{min}$. The mean rate of magazine entry during the stimulus receiving the unchanged outcome during compound training was $8.1 / \mathrm{min}$; that during the stimulus receiving the changed outcome was $6.9 / \mathrm{min}$. These two rates were not reliably different $[T(15)=45.5$, n.s.; $S E M s=1.07,0.94]$. 


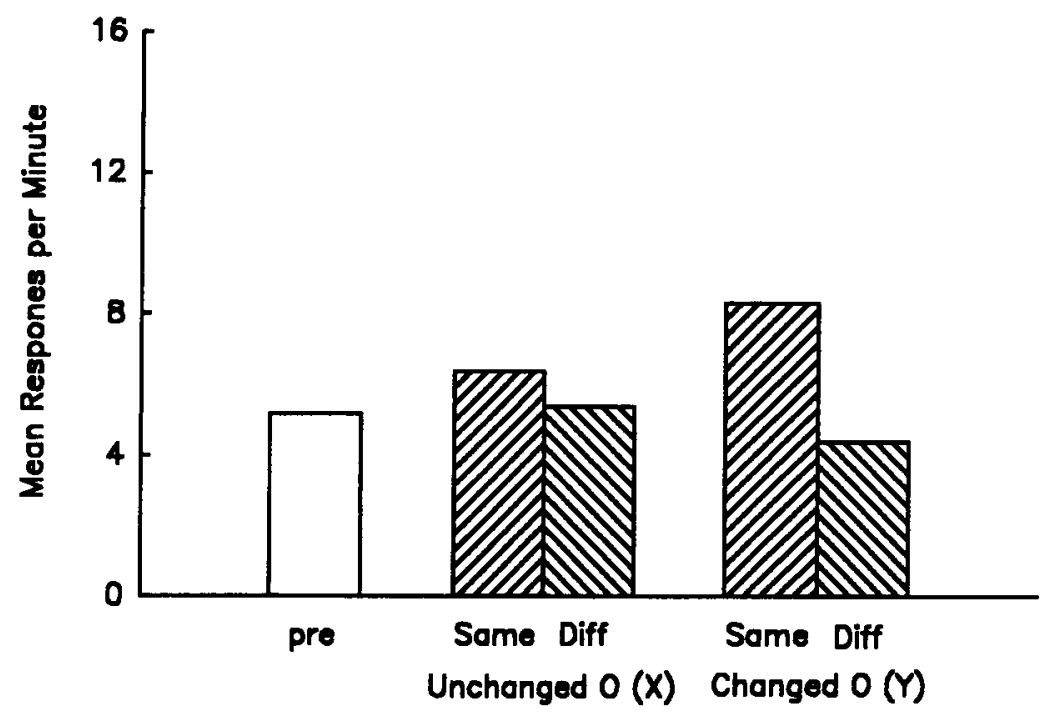

Figure 7. Test results of Experiment 3. Responding is shown during the prestimulus period and then for the response which had previously earned the same or a different outcome from that which reinforced the stimulus in compound. For one stimulus $(X)$, that outcome was unchanged from that used with its separately presented visual stimulus. For the other $(Y)$, the outcome was changed from that used with its separately presented visual stimulus. The $X$ and $Y$ stimuli were a noise and tone, counterbalanced.

These results suggest that there was comparable conditioning, at a low level, for these two stimuli. There is no evidence of greater responding for the stimulus that experienced a change in the outcome.

Figure 7 shows the results of instrumental responding during the transfer test. That figure displays the mean response rate in the absence of any stimulus, during a stimulus reinforced with the same outcome as that earned by the response, and during a stimulus reinforced with an outcome different from that earned by the response. These are displayed separately for the stimulus reinforced in compound with a changed and unchanged outcome. The patterns of data were quite different for the two stimuli. The stimulus trained with a changed outcome showed the characteristic differential elevation of the response with which it shared an outcome that has been observed elsewhere (e.g., Colwill \& Rescorla, 1988). For that stimulus, the rate was reliably greater on the same-outcome response than it was on the different-outcome response $[T(13)=6, p<$ $.01 ; S E M \mathrm{~s}=1.07,0.77]$. By contrast, there was not a reliable difference between elevation of same-outcome and different-outcome responses by the stimulus subjected to blocking with an unchanged outcome $[T(15)=31 ; S E M \mathrm{~s}=$ $1.19,0.94]$. The interaction, as measured by the greater same-different discrepancy for the changed-outcome stimulus compared with that for the unchanged-outcome stimulus, also proved reliable $[T(12)=14, p<.05]$.

This pattern of results suggests that changing the outcome over the course of a blocking procedure had little impact on performance of the Pavlovian magazine response. The relatively low and similar levels of responding elicited by the stimuli replicate the findings of Experiments 1 and 2.
They suggest that little conditioning was obtained whether or not the outcome was changed. However, the results of the transfer to the instrumental responses suggest that the underlying associative structures of the two stimuli were quite different. The stimulus for which the outcome was changed had apparently developed a sufficiently strong association with its outcome to govern differential transfer. The stimulus receiving a conventional blocking procedure with an unchanged outcome did not acquire that association nearly as strongly. These results imply that changing the outcome disrupted the blocking of associative learning despite the low level of performance of the Pavlovian magazine-approach response.

\section{EXPERIMENT 4}

The results of Experiment 3 encourage the view that changing from one outcome to another, equivalently valued, outcome during a Pavlovian blocking experiment may, in fact, produce some new learning. Experiment 4 was a systematic replication of that experiment in the context of instrumental learning. The ability of prior conditioning of $\mathrm{A}$ to produce blocking on the $\mathrm{AX}$ trials has been shown to extend to the case of instrumental discriminative conditioning, when $A$ and $X$ signal the opportunity of instrumental responding to produce a valued outcome (e.g., Williams, 1994). Moreover, prior data suggest that instrumental discriminative stimuli that signal the availability of different outcomes show excellent transfer of their control to new responses that have earned those outcomes (e.g., Colwill \& Rescorla, 1988). It is therefore of interest to ask whether the pattern of results observed in 
Experiment 3 will also occur within that instrumental paradigm.

The procedure of this experiment was similar to that of Experiment 3, with several noteworthy exceptions. First, the visual stimuli and the visual-auditory compounds signaled that making an instrumental response (nosepoke) would earn one or another outcome, rather than signaling the simple Pavlovian delivery of the outcome. Second, the success of conditioning was measured both in terms of transfer to new instrumental responses (lever and chain) and in terms of differential control of nosepoking; however, these were assessed in separate sessions. Third, a somewhat different procedure was used to implement a within-subjects design for changing the outcome for one compound and not for another. In Experiment 3 , the visual stimuli signaled different outcomes during initial conditioning but the compounds signaled a common outcome. In Experiment 4, the same outcome was used for both visual stimuli during initial conditioning but the compounds signaled the availability of different outcomes. Fourth, the separate trials with the visual stimuli given during compound training in Experiment 3 were deleted from Experiment 4.

\section{Method}

\section{Subjects and Apparatus}

The subjects were 16 male rats of the same sort and maintained in the same way as in previous experiments. The apparatus was eight operant chambers identical to those used in previous experiments, except that the flashing-light stimulus was mounted not on the ceiling but at floor level outside the rear of the chamber.

\section{Procedure}

Response training. Initial magazine and response training were given as in Experiment 3. After the training with the lever and chain, all animals were trained to nosepoke. For half of the animals, the reinforcer used for nosepoke was pellets; for the other half of the animals it was sucrose. Then all animals received 5 days of VI training, with responding on the lever and chain earning different outcomes, in the manner of Experiment 3 . They then received a day containing a single 20 -min VI session with the nosepoke, using the same outcome as in initial training.

Discrimination training. On each of the next 12 days, the animals received discrimination-training sessions with the nosepoke response. Each session contained 16 presentations each of a $30-\mathrm{sec}$ houselight (L) and a 30-sec flashing light (F). During these stimuli, nosepoking resulted in reinforcements according to a VI 30-sec schedule. For half of the animals, responding during both stimuli led to pellets; for the other half of the animals, responding led to sucrose. The ITI was variable around a mean of 30,60 , and $90 \mathrm{sec}$ for the first 3 days of training, respectively. Thereafter, the mean ITI was $2 \mathrm{~min}$, yielding a session duration of $64 \mathrm{~min}$.

Compound conditioning. On the next 3 days, all animals received discrimination training of the same sort as in the previous phase, except that the $1800-\mathrm{Hz}$ tone $(\mathrm{T})$ accompanied $\mathrm{L}$ and the noise $(\mathrm{N})$ accompanied $\mathrm{F}$. The outcomes earned in the different compounds differed such that for one compound it was the same as that earned during both $\mathrm{F}$ and $\mathrm{L}$ and for the other compound it was different from that previously earned during $\mathrm{F}$ and $\mathrm{L}$. This was counterbalanced across the identities of $\mathrm{T}$ and $\mathrm{N}$. All trials were 30 -sec long, and the ITI was variable around a mean of $2 \mathrm{~min}$.
Response retraining. On the next 2 days, the animals received retraining and extinction with the lever and chain. On the first of these, they received two VI sessions in the manner of prior training. On the second, they received two 8-min extinction sessions, one with lever and one with chain.

Test. On the next day, the animals received two test sessions in which the auditory stimuli were presented, one with lever available and one with chain available. Each 8 -min session contained four 30 -sec presentations each of $\mathrm{N}$ and $\mathrm{T}$, in counterbalanced order, delivered with an interstimulus interval of $30 \mathrm{sec}$. On the following day, the animals received a similar test of $\mathrm{N}$ and $\mathrm{T}$ but with only the nosepoke present.

\section{Results and Discussion}

Initial VI and discrimination training proceeded without incident. On the final day of VI training, the mean response rate during the lever and chain was 8.8 responses/ min. On the final day of discrimination training, the mean response rates were 30.4 and 2.5 during the stimulus and prestimulus periods, respectively. Responding was similar during the compounds receiving a changed or unchanged outcome. On the final compound day, the mean responses rates were 29.8 and 1.3 , during the compound and prestimulus periods, respectively.

Figure 8 shows the results of the test sessions in which $\mathrm{N}$ and $\mathrm{T}$ were presented while the animal had the opportunity to leverpress and chainpull. The results are shown combined across all the prestimulus periods, and for the response that had earned either the same or different outcome from that earned by the auditory stimulus in compound training; they are shown separately for the stimulus whose outcome was unchanged from that which its visual stimulus signaled and the stimulus whose outcome was changed. Both stimuli elevated responding relative to the prestimulus period. However, the patterns of elevation were quite different. The stimulus that had earned an outcome different from what its visual stimulus had signaled showed differential elevation of the two responses, augmenting more the response with which it shared an outcome than it did the other response $[T(15)=$ $23.5, p<.05 ; S E M \mathrm{~s}=2.62,3.64]$. By contrast, the stimulus receiving a standard blocking procedure with an unchanged outcome showed no differential elevation. The difference between these two results also proved reliable $[T(15)=25, p<.05]$. These results suggest that the first stimulus had acquired a stronger association with its outcome than had the second stimulus.

The test of responding to the nosepoke with the noise and tone, given on the next day, showed little evidence of differential blocking. During that session, the mean rate during the prestimulus period was $2.4 / \mathrm{min}$. The response rates were 5.2 and 6.1 during the stimulus receiving a changed outcome and one receiving the same outcome, respectively. The rates during the two stimuli were not reliably different from each other.

These results are quite similar to those of Experiment 3. Despite the fact that changing an outcome in the course of a blocking procedure had relatively little impact 


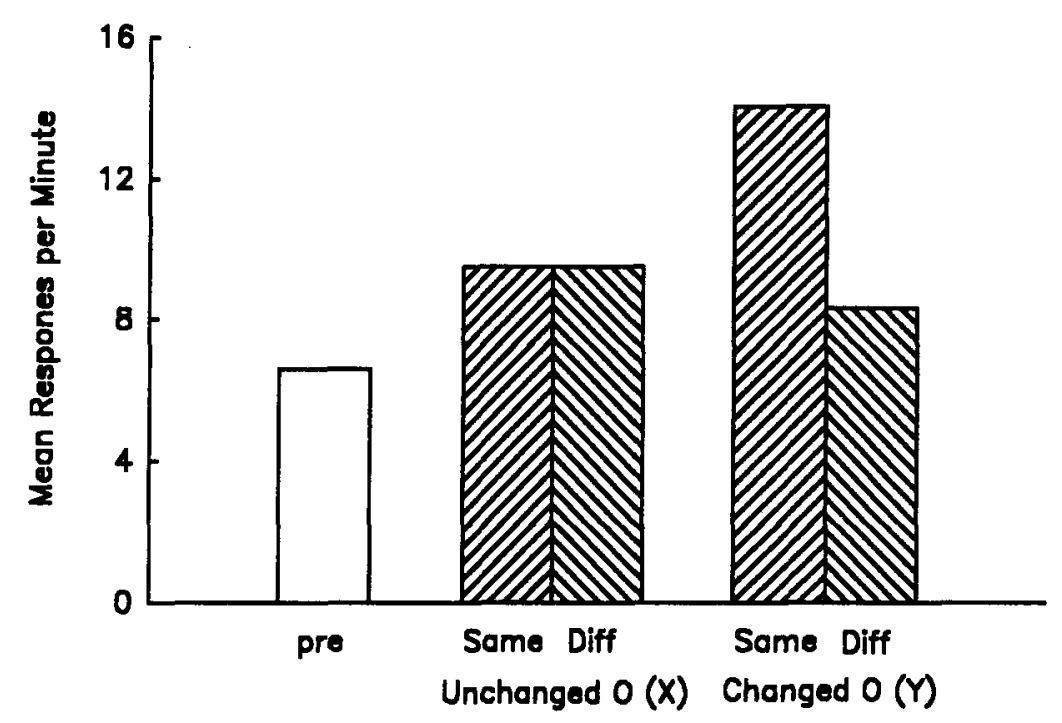

Figure 8. Test results of Experiment 4. Responding is shown during the prestimulus period and then for the response which had previously earned the same or a different outcome from that which reinforced the stimulus in compound. For one stimulus $(X)$, that outcome was unchanged from that used with its separately presented visual stimulus. For the other (Y), the outcome was changed from that used with its separately presented visual stimulus.

on the amount of conditioning observed for the originally trained response, transfer showed evidence of substantially different amounts of learning about the outcomes.

\section{GENERAL DISCUSSION}

These experiments show clear evidence for robust blocking of conditioning in a magazine-approach preparation. Blocking occurred whether the same outcome was used throughout the experiment or qualitatively different outcomes were used following the element and the compound. Moreover, the results of Experiments 3 and 4 suggest that the magnitude of the conditioning was comparable under those two procedures, when measured in terms of the originally trained response. However, those experiments also suggested that changes in the outcome had an important effect on the underlying associative structures that generated that performance. Transfer procedures gave evidence that when the outcome that followed the BY compound was different from that which followed $B$ alone, then $Y$ acquired an association with that new outcome. By contrast, when the same outcome followed AX and A alone, then X seemed to develop little by way of an association with that outcome. Apparently the similar absence of performance of the original response during $\mathrm{X}$ and $\mathrm{Y}$ occurred despite their acquiring different amounts of information about the outcomes that followed them.

Of course, it is hazardous to make direct comparisons between the original magazine and nosepoke responses on the one hand and the transfer responses on the other. It is quite possible that these responses differ in their in- herent sensitivities to associative changes. This is particularly true in Experiment 3, in which the transfer measure involved a choice whereas the magazine approach involved the simple likelihood of responding. Moreover, it is possible that, in Experiment 3, the concurrent presence of the transfer responses interfered with the magazine response, although earlier work suggests that associative changes are easily detected in the magazine-approach response with this concurrent measurement procedure (e.g., Rescorla, 1999). Although Experiment 4 avoided using both concurrent measurement and a choice test, it also conducted the test of the original response only after the transfer test. That prior transfer test may have reduced the sensitivity of the nosepoke response. Nevertheless, the numerically greater occurrence of the original response to $\mathrm{X}$ rather than to $\mathrm{Y}$, both Experiments 3 and 4 provide little support for the view that changing the outcome disrupted blocking for the original magazine and nosepoke responses. What does seem clear is that changing the outcome or not yielded quite different patterns of transfer, suggesting the presence of different underlying associative structures. Apparently some greater learning occurred with a procedure that made $Y$ informative by making a qualitative change in the outcome when $\mathrm{Y}$ was introduced.

One interpretation of these results can be constructed from a view suggested by Konorski (1967). That view emphasizes that different outcomes have multiple components, some shared with each other and some unique. One might then imagine that when $\mathrm{O} 1$ is the reinforcer for $\mathrm{B}$ and $\mathrm{O} 2$ is the reinforcer for $\mathrm{BY}, \mathrm{Y}$ would be blocked from learning the components shared by $\mathrm{O} 1$ and $\mathrm{O} 2$ but not blocked from learning about the components unique 
to $\mathrm{O} 2$. To the degree that the same conditioned response is being measured when $\mathrm{O} 1$ and $\mathrm{O} 2$ are used, it is plausible to think that it is the shared features of those outcomes that govern the shared response (i.e., magazine approach and nosepoking). In that case, one might well expect to see blocking when behavior is measured in terms of that shared response but not when it is measured in terms of responses controlled by the unique features of $\mathrm{O} 1$ and $\mathrm{O} 2$. This view of blocking was suggested briefly by Bakal et al. (1974). A much more well-developed version has been articulated by Wagner and his collaborators (e.g., Betts et al., 1996). According to the those authors, outcomes might share important affective properties but differ in their sensory features. They found evidence to support that position, measuring startle and eyelid responses in rabbits. It is not too difficult to suppose that the pellets and sucrose used here share a positive affective property but have distinctive sensory features. If one further supposed that the magazine approach was governed by the affective properties but the transfer to instrumental responses mediated by shared outcomes was based on sensory properties, then the results of Experiment 3 would be fully expected. If one likewise assumes that the nosepoke response of Experiment 4 depends on the affective properties of the stimuli, an account of that experiment can also be given.

This kind of interpretation can also be applied to overexpectation experiments in which two previously conditioned stimuli are presented together and reinforced. Even if those stimuli have been paired with positively valued outcomes having different sensory properties, one would anticipate summation of the affective components. If the total affective value of the compound exceeded that of the single outcome's actually occurring, then one would expect an undermining of the affective component of each of the stimulus elements, despite the preservation of their associations with the sensory components of the outcomes. That is, one would anticipate seeing a reduction in the original response controlled by the component stimuli at the same time that one observes successful transfer to instrumental responses based on the sensory features of the outcomes. Just this outcome has recently been observed for stimuli and outcomes like those used here (Rescorla, 1999).

What is less clear is the nature of the process by which the affective component is diminished. It is possible that it represents either a loss of the originally learned affect or a superimposition of some other outcome-independent affective process. A natural candidate for the latter process would be frustration resulting from value of the outcome anticipated being greater than the value of that actually obtained. If frustration were a consequence of the affective value conditioned to a stimulus, and relatively independent of the conditioned sensory features, then its development would leave intact information about identities of the outcomes. Moreover, its superimposition on an intact initial learning could provide a mechanism for changes in performance with time, such as the sponta- neous recovery from extinction or from the decremental effects of replacing one outcome by another. Frustration might also become conditioned to the particular responses that occur prior to its evocation, thus providing a means for understanding the effects of response specificity of depression observed in earlier experiments (e.g., Rescorla, 1993).

An alternative way of viewing different outcomes is as configural events, analogous to the description which is sometimes given of signals (e.g., Pearce, 1987). In that view, each outcome might be a separate event, to be learned about individually. However, outcomes may also be similar to each other in the affective and behavioral responses that they evoke. Then the changing from $\mathrm{O} 1$ to $\mathrm{O} 2$ as the consequent for a stimulus would necessitate learning about the entire $\mathrm{O} 2$ event. If, as earlier results suggest, this does not concurrently result in the loss of the association with $\mathrm{O} 1$, one might expect that the total response to the stimulus would be inappropriately large. This could result in a depressive mechanism which would diminish responding, perhaps by the conditioning of frustration, in the manner envisioned above. On a view like this, a shift in outcome during the course of a blocking experiment would produce the learning about the new configural $\mathrm{O} 2$ as well as the development of some depressive mechanism.

There is little in the present data to choose between these alternative conceptualizations of outcomes. However, an elemental and configural view of the outcomes may provide somewhat different descriptions of the trialto-trial dynamics that result from changing the outcome. On an elemental view, replacing $\mathrm{O} 1$ with an $\mathrm{O} 2$ introduces new sensory features but leaves any shared features unchanged. Consequently, there will be new learning about the sensory features but not necessarily any new learning about the shared (affective) features. On the other hand, a configural account can be seen as anticipating new learning about the whole $\mathrm{O} 2$ event, with the result that the common affective and behavioral responses which $\mathrm{O} 1$ and $\mathrm{O} 2$ control will also grow. In that case, those common responses would be inappropriately large and could be seen as engaging an outcome-independent depressive process to bring them into line. Consequently, a configural account might involve not simply the failure to learn about the common features but instead the development of an active depressive process that overcomes the additional learning about those features that pairing the stimulus with $\mathrm{O} 2$ produces.

This general line of thinking has been useful in interpreting experiments in which a single stimulus is first paired with $\mathrm{O} 1$ and then with $\mathrm{O} 2$. It has led to the expectation that the same depressive process occurs under those circumstances as when a stimulus is first paired with $\mathrm{O} 1$ and then nonreinforced. That, in turn, has led to the prediction that one might observe similar changes over time in responding to a stimulus under the two cases. That is, it has led to the expectation of a spontaneous-recovery-like process when a stimulus is first paired with $\mathrm{O} 1$ and then 
with O2. In a variety of experiments (e.g., Rescorla, 1997a, $1997 b)$ that prediction has been confirmed. Whether a similar result occurs in blocking experiments remains to be seen.

The results and thinking described here help to provide some understanding of the processes that occur when qualitative changes are made in the outcomes during a blocking procedure. But it is not clear to what degree they are helpful in dealing with results from changes in the quantitative properties of a single outcome. The primary implication of the present discussion is that in order to understand the results of a blocking experiment, it is important to use dependent variables that are sensitive to the detailed identities of the outcomes being associated. To the degree that outcomes differing along quantitative dimensions are viewed as qualitatively different, these results recommend assessment procedures that can detect those differences.

In any case, the present experiments suggest that qualitative changes in the outcome in the course of a blocking experiment do not go unnoticed by the organism. There is substantial learning about the newly introduced outcome which, although it may not be exhibited in the originally trained response, can be detected by transfer procedures.

\section{REFERENCES}

Bakal, C. W., Johnson, R. D., \& Rescorla, R. A. (1974). The effect of change in US quality on the blocking effect. Pavlovian Journal of Biological Science, 9, 97-103.

BetTs, S. L., BRANdon, S. E., \& WaGner, A. R. (1996). Dissociation of the blocking of conditioned eyeblink and conditioned fear following a shift in US locus. Animal Learning \& Behavior, 24, 459-470.

Blaisdell, A. P., Denniston, J. C., \& Miller, R. R. (1997). Unblocking with qualitative change of unconditioned stimulus. Learning \& Motivation, 28, 268-279.

Colwill, R. M., \& Rescorla, R. A. (1985). Post-conditioning devaluation of a reinforcer affects instrumental responding. Journal of Experimental Psychology: Animal Behavior Processes, 11, 120-132.

Colwill, R. M., \& Rescorla, R. A. (1988). Associations between the discriminative stimulus and the reinforcer in instrumental learning. Journal of Experimental Psychology: Animal Behavior Processes, 14, 155-164.

Cotton, M. M., Goodall, G., \& Mackintosh, N. J. (1982). Inhibitory conditioning from a reduction in the magnitude of reinforcement. Quarterly Journal of Experimental Psychology, 34B, 163-180.

Delamater, A. (1996). Effects of several extinction treatments upon the integrity of Pavlovian stimulus-outcome associations. Animal Learning \& Behavior, 24, 437-449.

Dickinson, A., Hall, G., \& Mackintosh, N. J. (1976). Surprise and the attenuation of blocking. Journal of Experimental Psychology: Animal Behavior Processes, 2, 313-322.

Ganesan, R., \& Pearce, J. M. (1988). Effect of changing the unconditioned stimulus on appetitive blocking. Journal of Experimental Psychology: Animal Behavior Processes, 14, 280-291.
Holland, P. C. (1984). Unblocking in Pavlovian appetitive conditioning. Journal of Experimental Psychology: Animal Behavior Processes, 10, 476-497.

HollaND, P. C. (1988). Excitation and inhibition in unblocking. Journal of Experimental Psychology: Animal Behavior Processes, 14, 261-279.

KAMIN, L. J. (1968). Attention-like processes in classical conditioning. In M. R. Jones (Ed.), Miami Symposium on the Prediction of Behavior: Aversive stimuli (pp. 9-32). Coral Gables, FL: University of Miami Press.

KONORSKI, J. (1967). Integrative activity of the brain. Chicago: University of Chicago Press.

MiLleR, R. R., \& MATZEL, L. D. (1988). The comparator hypothesis: A response rule for the expression of associations. In $\mathrm{G}$. H. Bower (Ed.), The psychology of learning and motivation: Advances in research and theory (Vol. 22, pp. 51-92). New York: Academic Press.

Pearce, J. M. (1987). A model of stimulus generalization for Pavlovian conditioning. Psychological Review, 84, 61-73.

Rescorla, R. A. (1992). Associations between an instrumental discriminative stimulus and multiple outcomes. Journal of Experimental Psychology: Animal Behavior Processes, 18, 95-104.

ResCoRLA, R. A. (1993). Inhibitory associations between $S$ and $R$ in extinction. Animal Learning \& Behavior, 21, 327-336.

ResCoRla, R. A. (1996). Preservation of Pavlovian associations through extinction. Quarterly Journal of Experimental Psychology, 49B, 245. 258.

ResCORLA, R. A. (1997a). Spontaneous recovery after Pavlovian conditioning with multiple outcomes. Animal Learning \& Behavior, 25 , 99-107.

ResCoRLA, R. A. (1997b). Spontaneous recovery of instrumental discriminative responding. Animal Learning \& Behavior, 25, 485-497.

RESCORLA, R. A. (1998). Instrumental learning: Nature and persistence. In M. Sabourin, F. I. M. Craik, \& M. Roberts (Eds.), Proceedings of the XXVI International Congress of Psychology: Vol. 2. Advances in psychological science: Biological and cognitive aspects (pp. 239257). London: Psychology Press.

RESCORLA, R. A. (1999). Summation and overexpectation with qualitatively different outcomes. Animal Learning \& Behavior, 27, 50-62.

Rescorla, R. A., \& Holland, P. C. (1982). Behavioral studies of associative learning in animals. In M. R. Rosenzweig \& L. W. Porter (Eds.), Annual review of psychology (Vol. 33, pp. 265-308). Palo Alto, CA: Annual Reviews.

WaGner, A. R. (1976). Priming in STM: An information-processing mechanism for self-generated or retrieval-generated depression in performance. In T. J. Tighe \& R. N. Leaton (Eds.), Habituation: Perspectives from child development, animal behavior, and neurophysiology (pp. 95-128). Hillsdale, NJ: Erlbaum.

Wagner, A. R., Mazur, J. E., Donegan, N. H., \& Pfautz, P. L. (1980). Evaluation of blocking and conditioned inhibition to a CS signaling a decrease in US intensity. Journal of Experimental Psychology: Animal Behavior Processes, 6, 376-385.

WATT, A., \& HONEY, R. C. (1997). Combining CSs associated with the same or different USs. Quarterly Journal of Experimental Psychology, 50B, 350-367.

Williams, B. A. (1994). Blocking despite changes in reinforcer identity. Animal Learning \& Behavior, 22, 442-457.

(Manuscript received March 18, 1998; revision accepted for publication August 12, 1998.) 